\title{
The Science Goals of the Constellation-X Mission
}

\author{
Nicholas E White* ${ }^{\mathrm{a}}$, Harvey Tananbaum ${ }^{\mathrm{b}}$, Kimberly Weaver ${ }^{\mathrm{a}}$, Robert Petre ${ }^{\mathrm{a}}$, Jay Bookbinder ${ }^{\mathrm{b}}$ \\ a. NASA Goddard Space Flight Center, Greenbelt, MD 20771 USA \\ b. Smithsonian Astrophysical Observatory, 60 Garden St, Cambridge, MA 02138 US
}

\begin{abstract}
The Constellation-X mission will address the questions: "What happens to matter close to a black hole?" and "What is Dark Energy?" These questions are central to the NASA Beyond Einstein" Program, where Constellation-X plays a central role. The mission will address these questions by using high throughput $\mathrm{X}$-ray spectroscopy to observe the effects of strong gravity close to the event horizon of black holes, and to observe the formation and evolution of clusters of galaxies in order to precisely determine Cosmological parameters. To achieve these primary science goals requires a factor of 25-100 increase in sensitivity for high resolution spectroscopy. The mission will also perform routine highresolution X-ray spectroscopy of faint and extended X-ray source populations. This will provide diagnostic information such as density, elemental abundances, velocity, and ionization state for a wide range of astrophysical problems. This has enormous potential for the discovery of new unexpected phenomena. The Constellation-X mission is a high priority in the National Academy of Sciences McKee-Taylor Astronomy and Astrophysics Survey of new Astrophysics Facilities for the first decade of the 21 st century.
\end{abstract}

Keywords: Observatories, X-rays, Spectroscopy, Dark Energy, Black Holes.

\section{INTRODUCTION}

The Constellation-X mission is a key element in NASA's Beyond Einstein program, aimed at addressing the questions "What is Dark Energy?" and "What happens close to the event horizon of a black hole?". These questions lie at the heart of resolving the basic physical processes that underlie Einstein's Theory of General Relativity and the connection of General Relativity to the standard model of Physics.

The existence of black holes is a prediction of General Relativity, and over the past two decades their existence has been well established by observations. Most recently Chandra has demonstrated that black holes are ubiquitous and provide a substantial fraction of the total energy output in the Universe. The observation of material falling into the event horizon provides an opportunity to test General Relativity in the strong gravity regime. A broad iron K line has been discovered, with the asymmetric double peaked shape predicted by strong gravity expected from material emitted from close to the event horizon. This is an important new X-ray probe to test General Relativity in the strong gravity limit, much closer to the event horizon than reached by discrete features seen in other wavebands.

The recent discovery that the expansion of the Universe is accelerating suggests the presence of a Dark Energy that makes up $70 \%$ of the mass-energy content of the Universe. This is of interest to both Cosmologists and fundamental physics alike because it may be the infamous Cosmological constant that Einstein included in General Relativity, or some form of evolving energy field that permeates the Universe. Because the importance of resolving the nature of Dark Energy it is important that independent methods are followed to determine its nature. An important approach is to use Clusters of Galaxies, whose evolution and properties provide precision Cosmological probes independent of the cosmological distance ladder (via the Sunayev-Zeldovich effect).

Constellation-X will extend our capability for high resolution $\mathrm{X}$-ray spectroscopy by 25 to 100 times. Its key goals are to determine the fate of gas falling into a black hole by tracking spectral features close to the event horizon, and to determine the nature of Dark Energy via observations of galaxy clusters. The mission is optimized for these challenges but also provides the ability to observe other objects with unprecedented sensitivity. Constellation- $\mathrm{X}$ will also e.g. trace the evolution of black holes with cosmic time by obtaining detailed spectra of faint quasars at high redshift and be able

\footnotetext{
*Nicholas.e.white@nasa.gov
} 
to search for spectral features from the surfaces of neutron stars, which could finally determine the properties of matter at nuclear density. Constellation-X will be to X-ray astronomy, what the Keck Observatory has come to mean for optical astronomy.

This paper will review the science goals of the Constellation-X mission, concentrating on how the mission will address the Black Hole and Dark Energy questions. A companion paper $^{1}$ provides details on the mission implementation approach.

\section{THE MISSION}

The 0.25 to $10 \mathrm{keV}$ band contains the $\mathrm{K}$-shell lines for all of the abundant metals (carbon through zinc), and the L-shell lines of many. The detailed X-ray line spectra are rich in plasma diagnostics which also provide unambiguous constraints on physical conditions in many different astrophysical settings. These spectral diagnostics are central to addressing the Beyond Einstein Black Hole and Dark Energy science questions. To reach the highest redshift clusters of galaxies and provide time resolved spectroscopy of the variable spectral features close to the black hole event horizon requires a factor of 25-100 increased sensitivity over current high-resolution X-ray spectroscopy missions.

The flow down of the science requirements translates to a telescope system with $3 \mathrm{sq} \mathrm{m}$ peak collecting area covering a broad energy band from 0.25 to $40 \mathrm{keV}$. This combined with high spectral resolution spectrometers (DE/E of 300 to 3000 ) utilizing a combination of gratings below $1 \mathrm{keV}$, and micro-calorimeter arrays up to $10 \mathrm{keV}$ provides the required spectral capability. A hard X-ray telescope system will provide the additional coverage up to at least $40 \mathrm{keV}$ and is required to define the underlying continuum. This corresponds to an effective (telescope plus detector efficiency) area of $1.5 \mathrm{~m}^{2}$ at $1 \mathrm{keV}, 0.6 \mathrm{~m}^{2}$ at $6 \mathrm{keV}$ and $0.15 \mathrm{~m}^{2}$ at $40 \mathrm{keV}$. Details of the current reference mission design and instruments are given in Figure 1, as well as in a companion paper ${ }^{1}$.

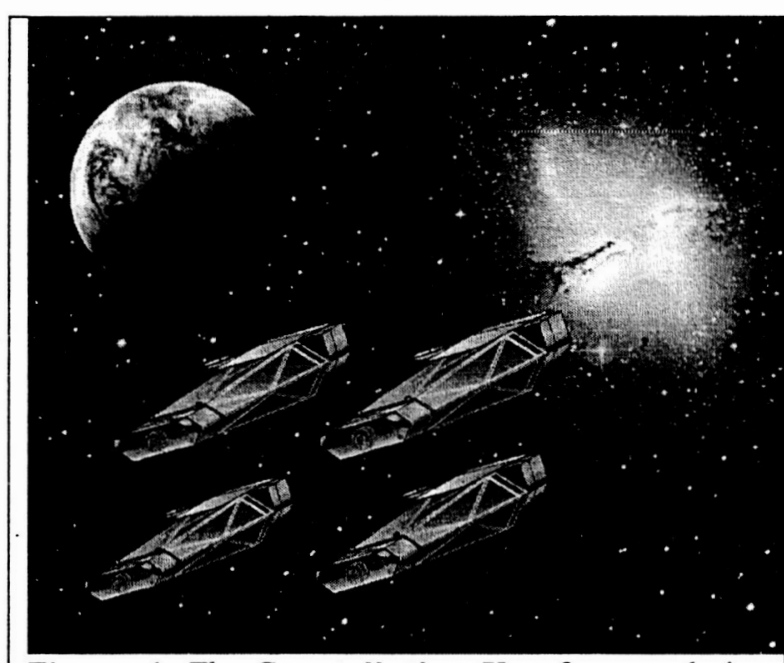

Figure 1. The Constellation-X reference design achieves high throughput and reduces mission risk by dividing the collecting area across four separate spacecraft launched two at a time. An orbit at L2 will facilitate high observing efficiency, provide an environment optimal for cryogenic cooling, and simplify the spacecraft design. Use of identical off-the-shelf spacecraft buses and a parallel production line will minimize cost.
Each satellite will contain two telescope systems: one with high energy resolution $(E / D E \sim 300-3000)$ for imaging X-ray spectroscopy $(0.2-10 \mathrm{keV})$, and one with low energy resolution $(E / D E \sim 10)$ for imaging hard $\mathrm{X}$ rays (to $60 \mathrm{keV}$ ). The spectroscopy telescopes will have 15 arcsec resolution (half power diameter) in a 2.5 arcmin field imaged by 900 -pixel quantum micro-calorimeters (with $2 \mathrm{eV}$ energy resolution). They will also include a set of reflection gratings (resolution 0.05 Angstrom in first order). The hard X-ray telescopes will be the first focusing optics above $10 \mathrm{keV}$ and have 1 arcmin resolution (half power diameter) in an 8 arcmin field.

All of the Constellation-X technologies are an evolution of existing, flight-proven instruments and telescopes. Substantial progress has been made in key areas of technology, including lightweight X-ray mirrors, improved energy resolution and construction of larger arrays of X-ray microcalorimeters, multilayer depositions for hard X-ray telescopes, and Cadmium-Zinc-Telluride detectors for hard X-rays. 
With these capabilities Constellation-X will be a major new astronomical facility. Constellation-X will also obtain with unprecedented sensitivity, precise measurements of the chemical composition and physical conditions of objects ranging from the closest stars to the most distant quasars via $\mathrm{X}$-ray spectroscopy.

The high priority of the Constellation-X mission and the science questions it is well suited to address have been recognized by the influential National Academy of Sciences McKee-Taylor survey of Astronomy and Astrophysics facilities for the first decade of the 21 st century, which ranked Constellation-X second only to JWST in large space based facilities. The more recent Academy Quarks to the Cosmos report of important science at the intersection of physics and astronomy also recognized the high priority of Constellation-X.

\section{DARK ENERGY}

Galaxy Clusters are the largest bound objects in the Universe, dominated by Dark Matter. The majority of the Baryons trapped in the gravitational grip of the Dark Matter is hot X-ray emitting gas. The evolution of Clusters of Galaxies is extremely sensitive to Cosmological parameters. They provide several independent methods to determine the dark energy and dark matter parameters

The X-ray brightness of clusters of galaxies scales as $(1+z)^{4}$ and current observatories can only reach redshifts for about 0.5 for detailed spectroscopy. Constellation- $X$ will have the ideal combination of collecting area and micro-calorimeter spectrometers to obtain detailed spectra of clusters at essentially any redshift where they exist (Figure 2). The measurable parameters are X-ray temperature and luminosity, which gives the cluster mass, the gas mass fraction (ratio of Baryons to total cluster mass (Baryonic plus Dark Matter), abundance and the velocity structure of the cluster. Constellation- $X$ will be able to measure the mass of any cluster of galaxy in the Universe $>10^{14}$ Solar masses within no more than a few tens of thousands of seconds. It will follow up to the planned microwave and X-ray galaxy cluster surveys

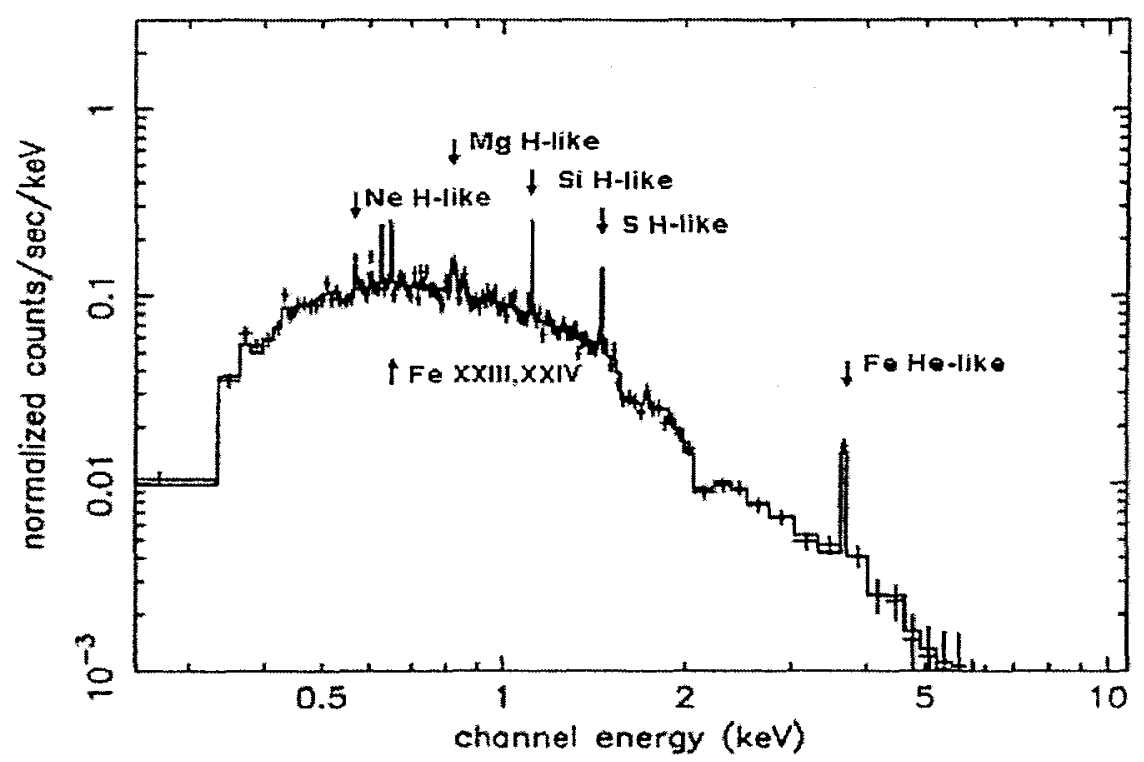

Figure 2. A simulated $50 \mathrm{ks}$ observation of a cluster of galaxies with an X-ray temperature of $4 \mathrm{keV}$ at $\mathrm{z}=0.8$. A Typc II supernova abundance distribution was assumed. The abundances are deternined to $10 \%$ accuracy for $\mathrm{Si}, \mathrm{S}$, and $\mathrm{Fe}$ and to $20 \%$ accuracy for $\mathrm{Ne}$ and $\mathrm{Mg}$

Constellation-X observers will apply at least three separate techniques to determine the Cosmological parameters, including the Dark Energy equation of state, w, 1) In combination with microwave background measurements use the 
Sunyaev-Zeldovich Effect (SZE) to measure the absolute distances to clusters, 2) Determine the gas to total mass fraction and use it as a "standard candle", and 3) Measure the evolution of the cluster parameters and mass function with redshift.

Great progress has been made recently in using the SZE to determine Cosmological parameters. This method of using the scattering of microwave photons off the hot cluster gas to determine the distance to the cluster requires precise microwave measurements of the decrement, that are now starting to become available. Combined with Chandra and XMM-Newton X-ray observations 41 clusters have been measured ${ }^{2}$ and have given a measure of the Hubble constant with uncertainties comparable to that obtained with the Hubble Space Telescope. The SZE error bars are dominated by systematic errors caused by having too few objects to average out geometric effects. With Constellation- $\mathrm{X}$ it will be possible to obtain precise measures for 500 clusters, to give the required precision. This will provide precise measures of Dark Energy equation of state (w) that are comparable to those being projected from other techniques ${ }^{3}$

Because Clusters of Galaxies are the largest objects in the Universe the relative fraction of dark matter to baryonic matter is expected to be constant. Imposing this to be the case provides a sensitive measure on the matter density of the Universe, thereby breaking a key degeneracy affecting other methods. Combining this with the determination of Cosmological parameters from the CMB removes the need for any external prior assumptions. Recently Allen et al $(2004)^{4}$ has used this technique to provide an independent verification of the Dark Energy parameters. With the many more clusters that will be observed at high spectral resolution with Constellation- $X$, it will be possible to provide precise constraints on $\mathrm{w}$ and also on the evolution of $\mathrm{w}$ with redshift. This will provide an important independent approach, and when combined with other techniques, greatly increase the precision.

Precision measurement of Cosmological parameters comes from the extreme sensitivity of the number of massive objects as a function of cosmic time and volume element. The distinction grows dramatically at higher redshift $(>0.5)$ and for the highest cluster mass. Chandra and XMM-Newton are limited to redshift $<0.8$ and only provide low spectral resolution $C C D$ spectra. Constellation- $X$ will accurately calibrate the mass-luminosity-temperature relation for large samples of high $(z>0.8)$ redshift clusters to significantly reduce statistical and systematic errors.

Observations of nearby clusters by Constellation- $X$ will verify our fundamental assumptions that the gas is in hydrostatic equilibrium and not dominated by turbulence or magnetic fields. The high spectral resolution will measure the line widths and thus limit turbulence and mass motion, while the hard $x$-ray imager will place limits on magnetic ficlds by searching for non-thermal high energy tails.

It is important to note that these measurements are independent of other techniques, give orthogonal error ellipses, and are physics based. These techniques are self-checking because one is using the same data set in multiple ways.

\section{BLACK HOLES}

A major science objectives of Constellation- $X$ is to observe relativistically broadened emission lines from Active Galactic Nuclei to determine masses and spins of their black holes. This is achieved by measuring both the detailed spectral shape of the line and its time variation. This will provide a precise clock to measure motion in the vicinity of the event horizon. The data will challenge our understanding of the behavior of matter within the framework of the general theory of relativity.

The discovery of relativistically broadened iron K lines by the ASCA observatory opened a new window to study black holes $^{5}$. The line originates from within a few gravitational radii of the black hole event horizon and as such probes much closer than spectral features seen in other wavebands. The more recent observations from XMM-Newton and Chandra have confirmed this feature exists and have shown tantalizing evidence for variability ${ }^{6}$

This iron $\mathrm{K}$ line can be utilized in several ways to observe the effects predicted by General Relativity. The line profile is very sensitive to the spin of the Black Hole, with the red wing being much more pronounced in the case of the Kerr spinning black hole (Figure 3). Turbulent processes within the accretion disk are expected to give rise to variability in the line on timescales of a few thousand seconds. This variability will take the form of narrow features that are blue and redshifted as the hot spot orbits and spirals into the black hole. 
Armitage and Reynolds $(2003)^{7}$ have made magneto-hydrodynamic simulations of accretion disk emission and the variations expected in the iron $\mathrm{K}$ line. They show that Constellation- $\mathrm{X}$ will provide the first detailed study of line variability. The mission will see the effects of non-axisymmetric structures orbiting the Black Hole. Constellation-X will follow the dynamics of individual blobs in the disk, and make a quantitative test of orbital dynamics in the strong gravity regime. Tantalizing evidence for such variations may have already been seen through a combination of XMMNewton and Chandra observations, but with insufficient area to follow these for several orbital cycles. Constellation-X will be able to utilize these features to map orbits of material close to the event horizon.

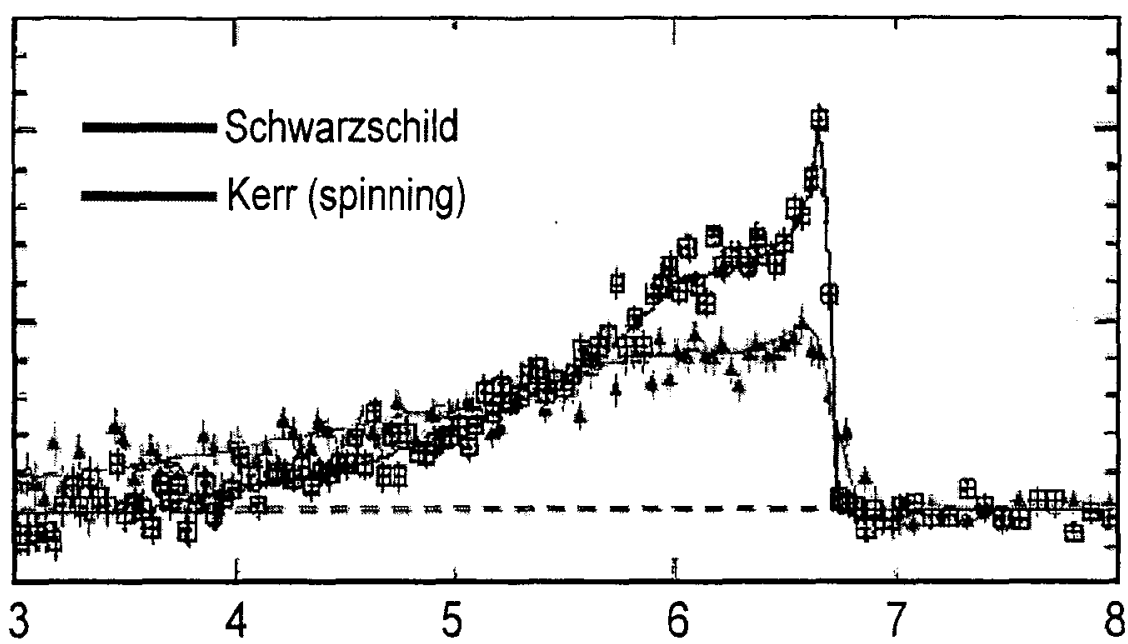

F.nerov

Figure 3. Simulations of the broad iron $\mathrm{K}$ line observed by Constellation-X observation for both a rotating (Kerr) and non-rotating black hole.

A second approach to using the iron $\mathrm{K}$ line to probe the strong gravity regime of General Relativity is to search for reverberation. AGN show dramatic flares, that may well be due to energy released in magnetic loops above the accretion disk, in a manner similar to coronal flares above the sun. It is to be expected that the iron line profile will change as the light echo sweeps across the disk. To search for this effect and utilize it requires the collecting area provided by Constellation- $X$.

Constellation- $\mathrm{X}$ will investigate how matter releases energy close to the event horizon. The brightness of the inner accretion disk can be inferred, to test models for energy release in accretion disks. Phenomena more exotic than accretion, such as the interaction of a spinning black hole with surrounding magnetized gas, can extract the black hole's energy of rotation. These processes can create the relativistic jets seen in many galactic nuclei, or pour tremendous power into the inner region of the accretion disk. Constellation- $X$ will give us the first detailed picture of these remarkable processes only hinted at by previous missions.

The dramatic increase in sensitivity of Constellation- $X$ will also allow us to trace the evolution of supermassive black holes in quasars and active galaxies. Constellation- $X$ will use the many black holes being found by the Chandra $X$-ray Observatory at high redshift to trace black hole evolution over cosmic time. The $\mathrm{X}$-ray band above a few keV is Iclatively free of ubscuration and thus aliows a ciear view of newiy born AGIN, even as they are shrouded by the young, dusty galaxies in which they reside. These observations will help determine the role of these black holes in the evolution of their host galaxies. 


\section{EQUATION OF STATE OF NUCLEAR MATTER}

An example of the rich facility class science that Constellation- $X$ will address is determining the equation of state of a nuclear matter in a neutron star. The physical constituents and equation of state of a neutron star remains a mystery after 35 yrs since their discovery. Constellation-X may finally provide the answers. The most promising approach is to use accreting systems, where the newly accreted material provides a source of metals to form spectral lines, before they rapidly sink into the neutron star atmosphere. Thermonuclear burning of this accreted material produces bring bursts of $\mathrm{X}$-ray emission and during these nuclear flashes the atmosphere of the neutron star shines brightly.

XMM-Newton observations of the burst source EX00748-676 has revealed evidence for discrete features from the neutron star photosphere ${ }^{8}$. These features required summing many bursts to detect and evolve through the burst. With Constellation- $X$ it will be possible to resolve these features in single bursts and follow their evolution. As discussed by Stromayer (2004) ${ }^{9}$ Constellation-X will also enable other approaches to constrain the equation of state using the pulsations observed by RXTE during the rise of the burst, whose amplitude and shape are very sensitive to the gravitational redshift at the surface of the neutron star.

\section{CONCLUSION}

The Constellation-X mission will usher in a new era in X-ray astronomy. The mission directly addresses the pressing questions on Black Holes and Dark Energy by enabling for the first time high throughput, high resolution $\mathrm{X}$-ray spectroscopy. By bringing the powerful spectral diagnostics that the $\mathrm{X}$-ray band has to offer into the astrophysics mainstream, Constellation- $X$ also address a broad range of topics ranging from the equation of state of nuclear matter, to the .creation of the elements in supernovae, and their distribution out into the intergalactic medium, to the evolution of black holes and their role in the formation of galaxies. These science goals have made the mission a high priority new astronomical facility called out in several National Academy of Science studies.

\section{REFERENCES}

1. Tananbaum et al 2004 (these proceedings)

2. Jones, M.R., et al 2004, submitted to M.N.R.A.S (see also Astro-ph/0103046)

3. Molnar,S.,Birkenshaw,M.\& Mushotzky,R. 2002 Ap J 573,91.

4. Allen et al 2004, M.N.R.A.S. in press

5. Tanaka, Y. et al 1995, Nature, 375, 659.

6. Turner, T.J., Kraemar,S.B., and Reeves, J.N. Ap.J. 2004, 603, 62.

7. Armitage,P.J. and Reynolds,C.S., 2003, M.N.R.A.S., 341, 1041.

8. Cottam, J., Paerels,F., and Mendez, M. 2002, Nature, 420, 51.

9. Strohmayer, T. Astro-ph/0403675 